ESJ Social Sciences

\title{
Chaussures à talons hauts et compensation phallique chez la femme
}

\author{
Manuela Naud Mvondo Meka \\ Université de Ngaoundere, Cameroon
}

Doi:10.19044/esj.2021.v17n27p242

Submitted: 25 November 2020

Accepted: 13 July 2021

Published: 31 August 2021
Copyright 2021 Author(s)

Under Creative Commons BY-NC-ND

4.0 OPEN ACCESS

Cite As:

Mvondo Meka M. N. (2021). Chaussures à talons hauts et compensation phallique chez la femme. European Scientific Journal, ESJ, 17 (27), 242.

https://doi.org/10.19044/esj.2021.v17n27p242

\section{Résumé}

Les chaussures à talons hauts étaient réservées aux bouchers, qui les arboraient pour se protéger du sang des animaux. Par la suite, elles deviennent l'apanage des bourgeois aristocrates qui les chaussent pour exprimer leur grandeur. Elles sont à chaque fois liées à l'expression d'une problématique spécifique. Malgré les différents inconforts et pathologies dont elles sont à l'origine, les chaussures à talons hauts sont devenues un élément incontournable de la mode féminine et un symbole de la féminité. L'objectif de cette étude est de déterminer la motion pulsionnelle profonde (inconsciente) qui pousse les femmes à arborer des chaussures qui, au-delà de la beauté visuelle dont elles font preuves, sont source d'un malaise physique bien réel. La psychanalyse appliquée a permis de mettre en évidence l'existence d'un sentiment inconscient d'infériorité chez la femme. La recherche d'une compensation phallique de cette infériorité ressentie est ce qui conduit au port des chaussures à talons hauts, en dépit des conséquences néfastes sur le corps. Une femme en talons hauts donne raison à Freud qui estime que toute la vie de la femme est une recherche du phallus.

Mots clés : Chaussures- Talons hauts- Femme- Phallus- Sentiment d'infériorité- Compensation phallique 


\title{
High Heel Shoe and Phallic Compensation in Women
}

\author{
Manuela Naud Mvondo Meka \\ Université de Ngaoundere, Cameroon
}

\begin{abstract}
High-heeled shoes were reserved for butchers, who wore them to protect themselves from the blood of animals. They subsequently became the prerogative of the bourgeois aristocrats who put on their shoes to express their greatness. They are each time linked to the expression of a specific problem. Despite the various discomforts and pathologies they cause, high-heeled shoes have become a staple of women's fashion and a symbol of femininity. The objective of this study is to determine the deep drive (unconscious) motion that prompts women to wear shoes which, beyond the visual beauty they demonstrate, are a source of very real physical discomfort. Applied psychoanalysis has brought to light the existence of an unconscious feeling of inferiority in women. The search for phallic compensation for this perceived inferiority is what leads to wearing high heels, despite the negative consequences on the body. A woman in high heels agrees with Freud who considers that the whole of a woman's life is a search for the phallus.
\end{abstract}

Keywords: Shoes- High heels- Woman- Phallus- Feeling of inferiorityPhallic compensation

\section{Introduction}

Une chaussure est un élément d'habillement assurant la protection du pied. Elle est un élément important de l'art vestimentaire. Elle rehausse la qualité de la tenue vestimentaire et fait ressortir la beauté du pied tout en le protégeant. Il existe plusieurs types de chaussures dont les chaussures à talons hauts.

Le talon haut est un type de talon, partie intégrante des bottes et chaussures, élevant significativement la hauteur du pied et dépassant alors celle des orteils. Le talon haut, en plus d'élever la taille de la personne qui le porte, donne généralement une illusion esthétique de jambes plus longues, plus fines et plus élancées. Le talon haut possède de nos jours une variété de styles, incluant notamment le talon aiguille.

D'après certains sites tels que ceux de Jimmy Choo et Gucci, un «talon bas » mesure moins de 6 centimètres ( 2,5 pouces), alors que les « talons moyens » mesurent $8,5 \mathrm{~cm}$ ( 2,5 et 3,5 po). Au-delà de 8 centimètres, le talon est considéré comme « talon haut ». (Talon haut, 2019, paragr.1) 


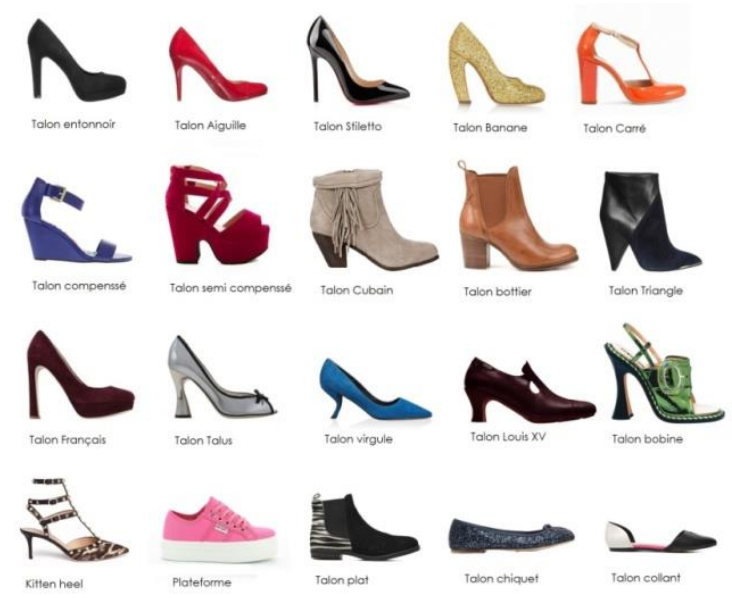

Figure 1 : Différents types de talons

http://kissmyshoe.com/wp-content/uploads/2014/11/Sans-titre5.jpg

La chaussure «n'est pas qu'un objet banal et insignifiant du quotidien, uniquement destiné à protéger les pieds des intempéries » (Audi, 2004, p.222). Tout comme la cravate (Mvondo, 2016), « la chaussure parle! Par ses formes, styles, couleurs et matériaux, la démarche qu'elle impose et le son qu'elle produit, la chaussure envoie une multitude de messages qui témoignent de l'individualité et de l'identité » (Audi, 2004, p. 222). Ainsi, « le soulier, c'est notre talon d'Achille. Il révèle notre personnalité, nos fragilités »(Psycho, 2017, paragr. 1). D'abord réservées aux hommes (Perez, 2018), les chaussures à talons hauts se sont progressivement imposées dans la mode féminine. Porter des chaussures à talons hauts apparait désormais comme un moyen d'exprimer et de défendre sa féminité, quoique ces chaussures ne soient pas confortables en tout point de vue et qu'elles soient très souvent cause de déformation et de maladie (Diallo, 2016).

Nous sommes prêtes à nous tordre de douleur pour entrer dans le " must have" du moment. Et les alertes des spécialistes n'y changent rien. Podologue, président de l'association Posturologie internationale, Philippe Villeneuve tire la sonnette d'alarme. "Les trois quarts de mes clients sont des femmes. J'ai beau les prévenir des risques, rien n'y fait." Pourtant, les talons de dix centimètres, les semelles compensées, les tongs... ont des effets très pervers sur notre corps. " La chaussure doit s'adapter au pied, et non l'inverse, insiste Philippe Villeneuve. Maux de tête, problèmes de dos et même de dyslexie... trouvent leur origine dans une mauvaise façon de se chausser (...). (Psycho, 2017, paragr. 9). 
Malgré ses multiples inconvénients, les chaussures à talons hauts restent un atout privilégié de la mode féminine (Modechaussure, 2021). «Trop de souffrance mais les femmes adorent » (Mani Bella, Facebook, 09/04/2021). Quelle est cette motion pulsionnelle latente (inconsciente) qui pousse les femmes à arborer ce type de chaussures qui, au-delà de la beauté visuelle dont elles font preuve, sont source d'un malaise physique bien réel? Cet article s'inscrit dans le champ de la psychanalyse appliquée (Freud, 1927/1933). Il vise à faire ressortir les sollicitations inconscientes qui sous-tendent un acte, une action, une production ou un comportement. Dans ce cas précis, il s'agit d'une réflexion théorique qui se donne pour objectif d'essayer de découvrir le sens et le contenu de la représentation des chaussures à talons hauts. En d'autres termes, il s'agit de découvrir la réalité psychique (latente, inconsciente) qui sous-tend le port des chaussures à talons hauts chez la femme. Pour ce faire, nous allons tout d'abord relater l'histoire des chaussures à talons hauts et ses conséquences sur le corps (cadre conceptuel). Par la suite, nous ferons appel à la théorie psychanalytique qui, à la lumière des travaux de Freud (1933, 1987, 2016) et d'Adler (1956), nous éclairera sur la femme et le sentiment d'infériorité (méthodologie et cadre théorique). Une analyse des pieds dans les chaussures à talons hauts et une discussion conduiront à la formulation d'une hypothèse explicative concernant les ressorts psychopulsionnels, qui favorisent le choix de ce type de chaussures chez la femme.

\section{Cadre conceptuel}

\subsection{Histoire des chaussures à talons hauts}

L'histoire des chaussures à talons hauts remonte à l'Egypte antique. À cette époque, elles étaient usitées par les bouchers, qui les portaient pour se protéger les pieds du sang des animaux tués dans la boucherie (LaPresse, 2019). Le métier de boucher étant à cette époque un métier exclusivement masculin, les chaussures à talons hauts étaient donc uniquement portées par des hommes. Outre les bouchers, elles étaient aussi portées par les cavaliers de l'empire Ottoman. Ces souliers leur permettaient de rester stables sur leurs étriers (Perez, 2018). Le roi Louis XIV voyait dans ces chaussures un moyen de magnifier sa grandeur. En effet, le roi Louis était une personne de petite taille (1m63); les chaussures à talons hauts lui offraient le moyen de se sentir grandi et d'affirmer sa grandeur. En 1533, sous l'influence de Catherine de Medecis, les chaussures à talons hauts deviennent un objet de luxe réservé aux membres de la haute société bourgeoise qui, par ces quelques centimètres de plus, exprimaient leur grandeur royale face aux autres populations (Cnew, 2016). En effet, « à la Cour du Roi Soleil, les nobles se hissent sur des talons d'au moins $12 \mathrm{~cm}$, d'abord pour se différencier du petit peuple - puisqu'ils sont surélevés, plus hauts, ils se rapprochent donc du Ciel et du Pouvoir Suprême » (VonGeschenk, 2015, paragr. 9). À cette époque, les chaussures à 
talons hauts étaient aussi bien portées par des femmes que par des hommes. En réalité, les chaussures à talons hauts étaient l'apanage des hommes. Lorsqu'elles deviennent un signe de bourgeoisie, elles sont désormais portées par les hommes et femmes de cette classe sociale. Par la suite, ces chaussures finissent par se démocratiser et n'importe qui peut désormais les arborer. Cette période semble correspondre au début de l'émancipation de la femme, qui est en fait une revendication de l'égalité avec l'homme. Ainsi :

Les femmes souhaitaient s'émanciper et commençaient à masculiniser leurs vêtements, épaulettes, cheveux coupés, c'est sans grande surprise finalement qu'elles ont ajouté les talons hauts à leurs atours. Leur garde-robe s'est ainsi rapprochée de plus en plus de celle des hommes. Au fur et à mesure on a vu se créer des différenciations concernant les types de talons, épais pour les hommes et fins pour les femmes. (Perez, 2018, paragr.6)

Au $19^{\mathrm{e}} \mathrm{S}$, on assiste à la :

"Grande Renonciation masculine". Ce terme a été créé par J.C. Flügel et désigne la période historique durant laquelle $[. .$.$] les hommes ont peu à peu renoncé à leur "sens$ de la mode" qu'ils ont relégué comme étant une "affaire de femmes". Les habits colorés et exubérants ont donc été remplacés par des habits plus sobres et les talons hauts sont ainsi presque devenus une exclusivité féminine. (Perez, 2018, paragr.6)

De nos jours, on observe une préférence marquée pour les chaussures à talon plat chez les hommes tandis que la chaussure à haut talon s'impose de plus en plus comme étant un symbole universel de la féminité. En somme «toute femme aime, adore, adule les chaussures à talons hauts, celles qui donnent la touche d'élégance, de glamour, de classe, à notre tenue. Celles qui nous rendent femme, en somme » (http://www.holala.ch/mode/1520.html cité par Tourre-Malen, 2011, p.731).

\subsection{Les conséquences du port des chaussures à talons hauts}

Il existe différentes conséquences du port des chaussures à talons hauts. Elles sont classées en conséquences dites positives et en conséquences dites négatives.

\subsubsection{Les conséquences positives}

Une conséquence positive est le résultat d'une justification positive. Elle renvoie à ce que les chaussures à talons hauts semblent apporter de positif 
à celles qui les arbore. Sont considérées comme conséquences positives : une taille rehaussée, une posture plus élancée, une illusion de jambes plus longues, une illusion de pieds plus petits, une illusion de doigts de pied plus courts, une meilleure définition des muscles des pieds, un renforcement du muscle fessier. En gros, tous ces avantages sont essentiellement esthétiques.

\subsubsection{Les conséquences négatives}

Les conséquences négatives renvoient aux différents inconvénients du port des chaussures à talons hauts. Avec une paire de chaussures à talons hauts, la partie supérieure du corps est projetée vers l'avant, ce qui a pour effet de modifier l'axe corporel. Pour compenser ce déséquilibre, les muscles du dos se rétractent et peuvent augmenter la lordose lombaire, la courbure naturelle du bas du dos, ce qui a pour effet de forcer autrement la marche. Par ordre de complication, il s'agit : des douleurs aux pieds, d'une démarche handicapante, d'une incapacité de courir, des changements au niveau des articulations, des risques élevés de fractures et d'entorses. "Des problèmes de rétractations musculaires, notamment au niveau des muscles du mollet ou du tendon d'Achille. [...] Ces fragilités peuvent être la cause de tendinites à partir du moment où l'on repasse sur des chaussures plates» (Benjamin, 2013, paragr.2). La compression constante des orteils et du pied est à l'origine des cals, cors et des ampoules.
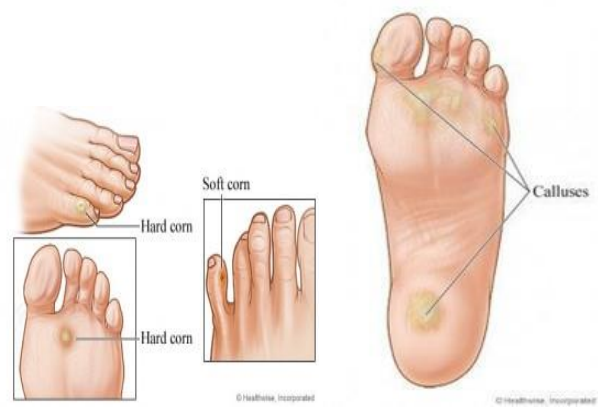

Figures 2 : illustration des pieds avec des cals, cors et ampoules https://topnaturalremedies.net/natural-treatment/natural-treatments-corns/

Cette compression constante est aussi à l'origine d'une déformation du pied et des orteils appelée hallux valgus. Il s'agit d'une déformation de l'avant pied d'origine héréditaire dont le processus pathologique est accéléré et accentué par le port des talons hauts (Benjamin, 2013). 


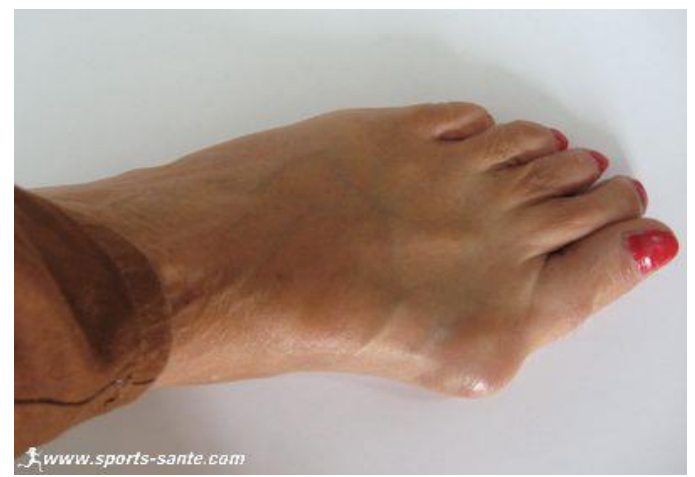

Figure 3 : Pieds atteint d'un hallux valgus

https://www.sports-sante.com/index.php/soin-des-pieds-l-hallux-valgus

La conséquence négative la plus grave est l'apparition d'un névrome de Morton qui peut aller jusqu'à paralyser la marche, vu la douleur occasionnée. Son traitement requiert une opération chirurgicale pour soulager le sujet de la douleur.
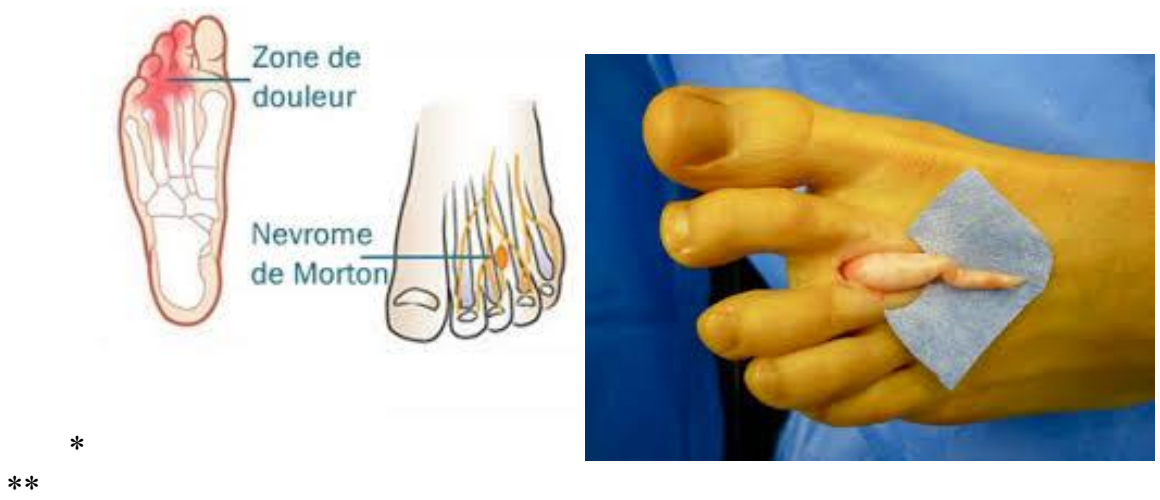

Figure 4 : Illustrations d'un Nevrome de Morton

*https://www.santescience.fr/symptomes/nevrome-de-morton;

**http://sante.orthodz.com/2016/07/20/nevrome-de-morton-causes-symptomes-ettraitement/

Malgré tous ces inconvénients, les femmes arborent toujours des chaussures à talons hauts. En effet, une grande majorité de femmes privilégie la mode, la séduction et l'esthétique au détriment du confort et du bien-être du pied (Heraldie, 2014). "Certaines accros n'hésitent pas à s'offrir un lifting du pied, des injections de collagène dans les talons ou une ablation d'orteil pour entrer dans les escarpins Jimmy Choo (...). La plupart des femmes préfèrent aller en enfer en talons hauts plutôt qu'au paradis en talons plats » (Psycho, 2017, paragr. 10). 


\section{Méthodologie}

La méthode utilisée pour répondre à notre question de départ est la psychanalyse appliquée. Elle utilise la théorie psychanalytique pour découvrir «le sens et le contenu de ce qui est représenté dans l'œuvre (Freud, 1927/1933, p.11). Il s'agit en fait d'exporter le savoir et les compétences de la psychanalyse à des domaines aussi variés que la poésie, la sculpture, la peinture (etc.). La psychanalyse appliquée analyse toute production de l'esprit pour nous en dévoiler la quintessence parfois inconnue de l'auteur lui-même. Elle nous dévoile le contenu inconscient qui motive une conduite, une création. Dans ce cas précis, le savoir et les compétences de la psychanalyse sont convoqués dans le domaine de la chaussure pour comprendre et expliquer pourquoi les femmes arborent des chaussures qui sont source d'une souffrance physique réelle plus ou moins significative. La psychanalyse appliquée fera ressortir le symbolisme des chaussures à talons hauts et la réalité inconsciente qui sous-tend leur port chez la femme.

Cette méthode ne nécessite pas une étude directe d'une population avec passation d'entretien, de questionnaire, de test ou autres. Elle vise l'analyse de ce qui est produit de façon brute et naturelle par l'esprit humain. L'analyse par Freud de l'œuvre de Shakespeare Hamlet, des exercices religieux (1932), du Moise de Michel-Ange (1933), et sa collaboration avec Einstein pour comprendre la guerre (1932) sont autant d'exemples de psychanalyse appliquée.

\section{Cadre théorique}

\subsection{La femme dans la théorie psychanalytique classique}

Dans la théorie psychanalytique classique, la différenciation sexuelle entre l'homme et la femme débute au stade phallique-œdipien. Avant ce stade, il existerait un seul et même sexe. En effet, l'enfant conçoit les êtres humains comme des personnes identiques, «il n'y aurait pas de différence anatomique entre les organes sexuels mâles et femelles » (Nasio, 1988/2001, p.19). Dans la pensée de l'enfant, tout le monde possède un seul et même organe sexuel qui serait le sexe masculin, donc le pénis. Il faut préciser qu'il ne s'agit pas du pénis en sa qualité d'organe sexuel masculin mais plutôt de sa représentation inconsciente manifestée par le phallus. Au total, deux phases sont primordiales dans le processus évolutif qui établit la différence sexuelle entre l'homme et la femme, à savoir la phase phallique et la phase œdipienne proprement dite.

\subsubsection{La phase phallique}

Le chemin qui conduit au complexe d'œdipe passe par la découverte des organes sexuels chez l'enfant. Lors de l'exploration de son corps, la petite fille se découvre un «petit pénis» (le clitoris en l'occurrence). Etant 
convaincue que les femmes adultes, y compris la Mère, possèdent un organe sexuel digne de ce nom, cela ne l'inquiète point. En effet, la Mère, celle qui prend soin de l'enfant depuis sa naissance, représente un symbole de puissance. Il est inconcevable pour l'enfant (qu'il soit garçon ou fille) de l'imaginer sans le phallus. Pour l'enfant, la Mère est un modèle de puissance confirmée. Il est donc impossible que l'enfant puisse la représenter sans attributs phalliques, cette Mère qui protège, rassure et maintient à bonne distance ses angoisses propres. Tout ceci changera, au détour d'une rencontre fortuite avec «le corps nu d'une femme, [elle] réalisera que sa Mère est elle aussi dépourvue de pénis» (Nasio, 1988/2001, p.22). Cette découverte est un choc qui contribue à détourner l'attention de la petite fille de la Mère vers le Père. Il s'agit là d'un changement d'objet d'amour rendu possible par la déception que la petite fille ressent à l'endroit de sa mère, une fois qu'elle a compris qu'elle est dépourvue de pénis ou encore que son clitoris (petit pénis) ne grandira jamais. En effet, « son amour s'adressait à une mère phallique et non à une mère châtrée. Il devient alors possible de s'en détourner et de laisser les sentiments hostiles, depuis longtemps accumulés, prendre le dessus » (Freud, 1933/1984, p.169). L'absence du pénis chez la petite fille est vécue comme une trahison de la Mère. Celle-ci l'aurait privée du phallus par simple jalousie, pour qu'elle soit châtrée comme elle et qu'elle souffre avec elle. «L'absence du pénis est ressentie comme un préjudice subi [que la petite fille] cherche à nier, compenser ou réparer » (Laplanche et Pontalis, 1967/2019, p.74,). Le complexe de castration ainsi mis en place va introduire la petite fille dans le complexe d'œdipe. Contrairement à ce qui se déroule chez le petit garçon, la petite fille doit effectuer un changement d'objet préalable pour vivre l'œdipe. Le manque, l'absence du pénis réussit à détourner la petite fille de son premier objet d'amour devenu mauvais (la Mère) vers un nouvel objet (le Père).

\subsubsection{Le complexe d'CEdipe et sa résolution chez la petite fille}

Le Père étant désormais son objet sexuel, la fillette projette ses désirs incestueux sur sa personne et l'amour primaire adressé à la Mère se transforme en haine. Elle espère que son nouvel objet d'amour (le Père) comblera le manque occasionné par la Mère. La libido autrefois investie sur le clitoris est déplacée dans le vagin et le « désir du pénis est remplacé par le désir d'avoir un enfant, ce dernier, suivant une vieille équivalence symbolique, devenant le substitut du pénis » (Freud, 1933/1984, p.155). L'envie de posséder l'organe (pénis) est transformée en une envie de posséder le porteur de l'organe (le Père). La petite fille entre ainsi dans le complexe d'œdipe proprement dit, à savoir : sentiments d'amour pour le Père et sentiments de haine pour la Mère.

La résolution du complexe d'œdipe chez la petite fille passe par la menace d'abandon que fait peser la Mère sur cette dernière si elle ne renonce 
pas à ses désirs incestueux vis-à-vis du père. La peur de perdre l'amour maternel semble être un motif de renoncement aux désirs incestueux. Seulement, cette angoisse n'apparait pas aussi puissante et terrifiante que l'est l'angoisse de castration. Ayant subi un premier abandon avec le sevrage au terme du stade oral, la petite fille ne prend pas au sérieux cette menace. En effet, même lorsque le sevrage est effectif, la Mère demeure auprès de la petite fille et continue de prendre soin d'elle. Une nouvelle menace d'abandon ne saurait donc mener à un renoncement aux désirs incestueux chez la petite fille. L'intervention du Père (c'est-à-dire d'une figure d'autorité) devient un impératif. Ce dernier devra travailler à la restauration de l'autorité de la Mère perdue par le fait de l'absence de phallus constatée chez elle par l'enfant. L'intervention du Père participe aussi à l'intégration des notions de loi et d'interdit à travers l'interdiction de l'inceste. La restauration de l'autorité de la Mère et l'interdiction de l'inceste conduiraient nécessairement à une dissolution du complexe d'œdipe. Ce qui faciliterait l'intégration des interdits parentaux (interdiction de l'inceste) et l'identification à la Mère.

Toutefois, La résolution effective du complexe d'œdipe chez la petite fille n'est pas évidente dans l'approche freudienne. Le complexe d'œdipe chez la fille dure toute son existence (Freud, 1905/1987). La femme est dépourvue de « pénis », ce qui signifie qu'elle ne possède pas le phallus. Son autorité doit donc sans cesse être restaurée, voire compensée. En effet, « la fille [demeure dans la situation œdipienne], pour une durée indéterminée, elle ne le démonte que tardivement, et lorsqu'elle le fait, c'est de manière incomplète » (Freud, 1920/2016 p.172). Selon la psychanalyse freudienne, l'absence/le manque ou l'envie du phallus serait à l'origine d'un sentiment d'infériorité (chronique) chez la femme. C'est ce dernier qui motive la recherche de compensation. Et comme le dit Adler (1911/1970, p.22) « le sentiment d'infériorité que tels ou tels organes inspirent à l'individu devient un facteur permanent de son développement, [de sa vie] psychique ».

\subsection{Le sentiment d'infériorité et sa compensation}

Tout sentiment d'infériorité conduit à la mise en place d'un processus de compensation visant soit à dépasser, masquer, soit encore à accentuer l'infériorité ressentie.

\subsubsection{Le sentiment d'infériorité}

L'Homme par définition est un être inférieur. Il doit sans cesse compenser cette infériorité pour s'adapter à son milieu de vie : « Le sentiment d'infériorité domine la vie psychique et on le trouve clairement exprimé dans les sentiments d'insuffisance, d'imperfection et dans les efforts ininterrompus fournis par les êtres humains » (Adler, 1933/1968, p.65). En psychanalyse, il ne renvoie pas toujours à une infériorité organique. Le sentiment d'infériorité 
est le résultat d'une incohérence entre désir et possibilité de satisfaction (plaisir). Ainsi, certains individus vont se forger un but final, purement fictif, caractérisé par la volonté de puissance pour pouvoir atteindre une possibilité de satisfaction, même si celle-ci est totalement détournée du plaisir réel voulu. Il se met donc en place dans le psychisme, un mécanisme appelé compensation. Il s'agit plus précisément d'une compensation psychique. Cette dernière sera développée par la femme, au détour de comportements spécifiques, pour accéder au phallus imaginaire. Ce mécanisme vient répondre au dommage de castration chez la jeune fille (Lagache, 1961).

\subsubsection{La compensation : Compensation psychique- Compensation phallique}

La notion de compensation a été développée la première fois par Claude Bernard (1865). Ce physiologiste de formation avait montré comment l'organisme parvient à compenser une infériorité organique en développant un peu plus les autres organes fonctionnels. C'est à Adler (1956) que l'on doit l'introduction de la notion de compensation en psychologie. Dans ce cas spécifique, il s'agit de compensation psychique. Elle est un :

Processus assimilable à l'homéostasie destiné à retrouver le niveau d'équilibre et de plénitude après une perte. La compensation agit comme un mécanisme de défense et d'adaptation à une situation affaiblissante. Dynamique de dépassement à l'origine de bien des progrès, si elle est positive et de bien des troubles si elle est négative. (Mormin et Viguier, 1993, p.165).

Adler (1956) a décrit deux groupes de compensations: les compensations négatives et les compensations positives. Les compensations positives sont des surcompensations. Elles correspondent en fait à des compensations réussies où le sujet a réussi à dépasser son infériorité. Les compensations négatives sont de deux types, à savoir les compensations dissimulatrices et les compensations exploratrices. Dans le premier type, le sujet masque son sentiment d'infériorité avec des comportements caractéristiques (manifester des comportements de supériorité par exemple). Dans le second type, le sujet utilise son infériorité pour capter l'attention de son entourage (utiliser son infériorité pour profiter des autres).

Une compensation positive est celle où le sujet parvient à faire de son infériorité une force qui lui permet de s'épanouir dans son milieu de vie interne et externe. Dans ce cas, le sujet parvient effectivement à surmonter son infériorité. Une compensation négative est celle où le sujet fait l'usage de mécanismes qui permettent juste de masquer son infériorité ressentie ou alors de l'exposer pour susciter de la pitié, de l'intérêt chez l'autre. Parfois même, 
le sujet peut aller jusqu'à présenter des comportements pouvant faire penser à une compensation réussie alors qu'il ne s'agit que d'un comportement traduisant l'exacerbation de son infériorité (c'est le cas dans le complexe de supériorité).

L'absence de pénis chez la femme est considérée comme étant un signe d'infériorité. En réalité, cette absence est à l'origine d'un sentiment d'infériorité qui favorise la mise en place d'un processus de compensation psychique. Cette compensation permet à la femme d'acquérir fantasmatiquement le phallus. Cette compensation est une compensation phallique parce qu'elle comblerait le vide phallique lié à l'absence du pénis.

\section{Analyses \\ 4.1. La place des pieds et des chaussures à talons hauts dans le processus de compensation phallique}

« Le pied est un symbole sexuel archaïque » (Freud, 1905/1987, p.64). Cela signifie qu'il est l'expression anatomique du pénis, ce pénis qui manque ou qui est absent chez la petite fille et chez la femme. La fameuse expression « troisième pied » chez les hommes en référence à leur pénis est très évocatrice de cet aspect. Freud (1905/1987, p.65) le rappelle en ces termes :

Le pied remplace le pénis de la femme, dont l'absence est si lourdement ressentie. [...] Dans bien des cas du fétichisme du pied, il a été possible de montrer que la pulsion scopique, dirigée à l'origine vers les parties génitales et qui cherche à accéder à son objet par en dessous a été retenue en route par l'interdit et le refoulement, moyennant quoi elle s'est attachée au pied ou au soulier qui ont pris valeur de fétiche. Conformément à l'attente enfantine, l'organe génital féminin fut dès lors représenté comme un organe masculin.

La chaussure ou « le soulier, la pantoufle, [sont un] symbole des parties génitales féminines»»(Freud, 1905/1987, p.64). «Freud a identifié la chaussure comme un symbole sexuel et le pied comme un symbole phallique. Or le pied se glisse dans la chaussure » (Heraldie, 2014, paragr. 3). Observons la radio ci-dessous d'un pied dans une chaussure à talon haut. 


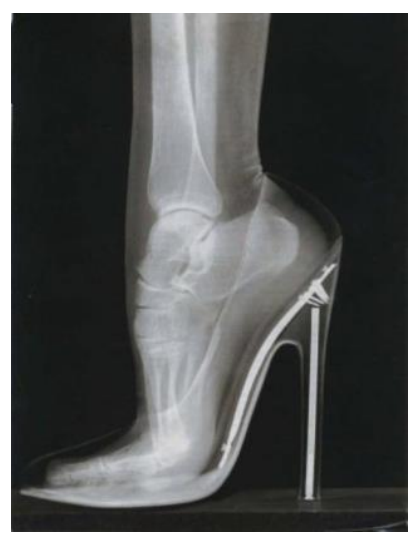

Figure 6 : Radiographie d'un pied dans une chaussure à talon haut (https://www.comment-economiser.fr/21-super-trucs-pour-chaussures-confortables.html)

Le pied bien tendu épouse parfaitement la forme de la chaussure. Un pied qui dompte la chaussure, une chaussure qui se laisse dompter et vis-versa, comme dans un coït entre deux personnes de sexes opposés consentantes. Dans ce sens, les chaussures à talons hauts sont comme une sorte d'enveloppe ayant pour finalité de compenser une fragilité issue de l'absence et/ou du manque du phallus (Freud, 1920/2016). Dans le même ordre d'idée, il existe un type de chaussures à talons hauts appelé "compensés ». En langage psychanalytique, cette appellation témoigne de l'existence d'un manque ou d'une infériorité ressentie.

Dans l'évolution de son histoire notamment, les chaussures à hauts talons se présentent comme étant un moyen de protection (chez les bouchers) et comme un moyen d'affirmer sa grandeur ou puissance (chez les bourgeois). Elles sont l'expression d'un besoin de protection (et de domination) chez les bouchers à qui elles permettent d'éviter d'être salis par le sang des animaux. Chez les bourgeois, elles répondent plutôt au besoin d'expression de leur grandeur. Dans les deux cas, les chaussures à talons hauts viennent aider le sujet à résoudre un problème (manifeste chez le boucher et latent chez les bourgeois). D'abord l'apanage des hommes, elles se sont féminisées dans un contexte d'émancipation de la femme qui revendiquait l'égalité avec les hommes. Faire comme un homme était donc l'exemple même d'une femme émancipée (Perez, 2018). Selon Adler (1930/1961, p.45) :

Le langage [que la femme en talons hauts] parle, si on sait le comprendre, montre à l'évidence que l'on est en présence d'un individu qui lutte pour que l'on reconnaisse sa valeur qui s'efforce actuellement d'obtenir à tout prix cette reconnaissance ; il est évident aussi qu' [elle] aspire sans cesse à une domination quasi divine sur son entourage pour échapper à son insécurité et à son sentiment d'infériorité. 


\subsection{Chaussures à talons hauts : expression d'une compensation dissimulatrice}

La compensation est une stratégie qui consiste à dissimuler ou à surpasser de manière consciente ou inconsciente nos désirs, faiblesses, sentiments d'insuffisance dans un secteur de la vie. Pour Freud (1905/1987), le pied de la femme est un substitut du pénis et la chaussure le substitut de ses organes génitaux. Ce pied mis dans une chaussure à haut talon symbolise un pénis en érection dans l'organe sexuel féminin en état d'excitation, de manque. Un mode de compensation fantasmatique traduisant l'existence d'un complexe d'infériorité. En effet, l'absence de phallus a plongé la petite fille dans une tristesse intense. Elle espérait retrouver ce dernier avec l'amour du Père, mais l'interdiction de l'inceste rend cette quête impossible (Freud, 1905/1987). Elle est donc contrainte de vivre sans le phallus. Ce dernier étant considéré comme un symbole de force et de puissance, son absence participe à l'installation d'un sentiment d'infériorité qui, avec le temps et le milieu, se cristallise en un complexe d'infériorité. Cette infériorité vécue est source d'angoisse et le Moi y fait face en développant une stratégie de compensation : la compensation phallique.

La compensation phallique désigne le fait d'utiliser des substituts pour pallier l'absence d'un phallus véritable (pénis fonctionnel). Dans ce cas précis, le sujet féminin adopte un comportement vestimentaire spécifique : le port des chaussures à talons hauts. Avec les chaussures à talons hauts, la femme se sent fantasmatiquement en pleine possession du phallus, bien qu'il ne s'agisse que d'un phallus imaginaire et totalement illusoire. Le port des chaussures à talons hauts correspond donc à une compensation dissimulatrice (compensation négative). Elle est négative parce que, bien qu'elle permette au sujet de fuir temporairement sa réalité psychique inconsciente par une possession illusoire du pénis, elle est source d'un inconfort physique réel. Et comme le dit l'artiste musicienne Mani Bella (Facebook, 8/11/20).), « les pieds souffrent énormément sur des talons aiguilles »

\section{Discussion}

L'histoire des chaussures à talons hauts montre que celles-ci, au départ, étaient un moyen de protection (utilisé par les bouchers) et qu'elles étaient uniquement portées par des hommes. Son avènement dans le monde de la mode avec les bourgeois aristocrates leur confère une fonction psychique à travers l'expression de grandeur revendiquée. En effet, dans le premier cas, les chaussures à talons hauts ont une fonction protectrice visible à l'œil (comme chausser des bottes pour se déplacer dans un environnement marécageux). Dans le second cas, elles ont une fonction psychique parfois imperceptible au premier abord (latente). Il s'agit de se montrer puissant pour voiler son impuissance ressentie. 
Ainsi, avec les chaussures à talons hauts, la femme expérimente la puissance phallique du pénis et même plus. Les propos de certaines actrices recensés par Marie Claire (s.d) vont dans ce sens. Roitfeld Carine affirme : « Portez toujours des chaussures à talons haut! Oui elles vous donnent du pouvoir »; Victoria Beckham déclare : «Je ne peux pas me concentrer avec des chaussures plates »; Sonia Rykiel s'interroge : « comment voulez-vous avoir un train de vie élevé sans avoir des hauts talons ?» Selon Christine Brad, «On reconnait aux femmes hauts perchées une forme de performance. Il y a dans leur geste une sorte d'excellence, qui leur confère une certaine autorité »; Vivienne Westwood pense que « les chaussures doivent avoir de très haut talons et des plateformes pour mettre la beauté des femmes sur un piédestal »; Brian Atwood dit : « la vie est courte, les talons ne doivent pas l'être ». Le pouvoir des talons hauts et leur caractère jouissif sont sans cesse mis en avant comme si le fait de les arborer est en lui-même une réparation des dommages de la castration. Plus loin encore, Adrienne, une star de téléréalité américaine affirme que «The higer the heel, the closer to God» [plus le talon est haut, plus l'on est proche de Dieu] («The american real houswives berverly hills », Saison 3, épisode 2, 2012-2013). Le concept de dieu est le symbole phallique suprême qui renvoie à celui qui détient la puissance absolue. Les chaussures à talons hauts sont donc incontestablement un substitut phallique, qui traduit l'existence d'un sentiment inconscient d'infériorité, lié au manque ou à l'absence du phallus (du pénis) chez la femme.

L'hypothèse théorique qui ressort de notre discussion est donc la suivante : «La femme porte des chaussures à hauts talons pour compenser une infériorité phallique due à l'absence du pénis ». "Avec des talons hauts, les femmes veulent voir et être vues » (Fresnel, 2016). Les chaussures à talons hauts sont une enveloppe destinée à compenser la fragilité de celle qui la porte. Cette fragilité résulte d'une absence du phallus ressentie depuis la période phallique-œdipienne. Avec les chaussures à talons hauts, la femme se sent en pleine possession du pénis et de puissance y afférente.

Certains estiment que les chaussures à talons hauts devraient être réservées aux personnes ayant une taille en dessous de la moyenne, ceci pour compenser le sentiment d'impuissance qu'elles peuvent ressentir face aux autres personnes de grande taille. On voit bien ici que c'est l'absence de quelques centimètres en plus qui oriente vers le choix des chaussures à talons hauts, justement pour compenser un ressenti d'infériorité y relatif. C'est exactement le même scénario chez les personnes ayant une taille moyenne (et plus) sauf que dans ce cas, le ressenti d'infériorité est psychique et totalement inconscient. En voulant exprimer à tout prix leur féminité, avec des chaussures à talons hauts, les femmes expriment un sentiment inconscient d'infériorité d'ailleurs très apprécié par les hommes. En effet, Guéguen (2015), chercheur 
en sciences du comportement à l'Université de Bretagne-Sud, a démontré dans une étude expérimentale que les femmes en hauts talons attirent deux fois plus d'hommes que les femmes en talons plats. Toujours dans cette même étude, il démontre que plus les talons sont hauts, plus les hommes sont attirés par les femmes qui les arborent.

\section{Conclusion}

Notre question de départ était la suivante : quelle est la motivation inconsciente qui pousse les femmes à arborer des chaussures qui, au-delà de la beauté visuelle dont elles font preuve, sont source d'un malaise physique bien réel ? Il était question de réaliser une analyse théorique relevant de la psychanalyse appliquée. Les travaux de Freud (1905/1987, 1920/2016) et Adler $(1930 / 1961,1911 / 1970,1956)$ constituaient notre base théorique d'analyse. Au terme de cette élaboration théorique, il ressort que la réalité psychique de la femme est dominée par l'absence et/ou le manque du phallus. Cette situation est vécue comme une situation d'infériorité qui pousse la femme à rechercher des mesures de compensation. L'une d'entre elles se trouve dans le port des chaussures à talons hauts. Il s'agit là d'une compensation dissimulatrice qui permet de masquer l'absence et/ou le manque du pénis (phallus). Avec les chaussures à talons hauts, la femme se sent symboliquement en pleine possession du pénis. Cette possession phallique est essentiellement fantasmatique et illusoire.

La compensation phallique dissimulatrice a de nombreuses conséquences négatives sur le corps de la femme, ce qui pour autant ne la dissuade pas de les arborer. Les chaussures à talons hauts se sont imposées progressivement comme un symbole exclusif de la féminité (de la mode féminine). Si l'on se réfère à la psychanalyse classique, être une femme c'est être en situation d'infériorité du fait de l'absence du phallus. Porter des chaussures à talons hauts traduit une recherche fantasmatique d'une puissance phallique absente. Une femme en talons hauts donne raison à Freud (1920/2016) qui estime que toute la vie de la femme est une recherche du phallus. Avec les chaussures à talons hauts, la femme espère « se libérer d'un sentiment de faiblesse afin d'atteindre les sommets, s'élever d'un « en bas » vers un «en haut» [...] devenir supérieur aux autres » (Adler 1930/1961, p.46). L'hypothèse théorique qui es découle est la suivante : «La femme porte des chaussures à hauts talons pour compenser une infériorité phallique due à l'absence du pénis».

Les chaussures à talons hauts devraient simplement être mises à l'écart des éléments de la féminité. La femme n'a pas besoin d'une illusion phallique masculine pour se sentir exister. Malheureusement, cette dernière demeure encore emprisonnée dans un modèle phallocratique qui fait du phallus (du pénis) un idéal à atteindre. La femme choisit donc de se laisser souffrir (porter 
des talons hauts) du moment que cette souffrance la rapproche un peu plus de l'illusion pénienne (du phallus). Un masochisme voulu pour accéder à une illusion phallique pathologique. "L'attachement des femmes à une technique dont elles sont les premières victimes offre une illustration de ce que Godelier (1982, p.60) cité par Tourre-Malen (2011, p.735), a appelé le "consentement des dominés à leur domination" ».

\section{References:}

1. Adler, A. (1949). Connaissance de l'homme. (J. Marty, Trad.). (Ouvrage initialement publié en 1927).

https://www.psychaanalyse.com/pdf/PHILO_BIBLIO_LIVRE_ADL ER_CONNAISSANC DE_L_HOMME_202PAGES.pdf

2. Adler, A. (1970). Le tempérament nerveux. (Trad., Roussel). Payot. (Ouvrage initialement publié en 1911.).

http://classiques.uqac.ca/classiques/adler_alfred/temperament_nerveu $\mathrm{x} /$ temperament.html

3. Adler, A. (1961). Pratique et théorie de la psychologie individuelle comparée. ((H. Schaffer, Trad.)Payot. (Ouvrage initialement publié en 1930.).https://psychaanalyse.com/pdf/psycho_indiv_comp_15_30.pdf

4. Adler, A. (1968). Le sens de la vie. Etude de psychologie individuelle. (H. Schaffer, Trad.). Payot. (Ouvrage initialement publié en 1933.) http://classiques.uqac.ca/classiques/adler_alfred/sens_de_la_vie/sens. html

5. Adler, A. (1956). La Compensation psychique de l'état d'infériorité des organes. Payot.

6. Audi, D. (2004). Compte rendu de [LAFORGE, VALÉRIE. Talons et tentations.Québec, Musée de la civilisation; et Montréal, Fides, 2001, 125 p., ill. ISBN2-7621-2375-5]. Rabaska, 2, 221-223. https://doi.org/10.7202/201669ar

7. Bagarry, B. (2014). Faveurs aux talons aiguilles. http://www.psychotherapeute-paris11.fr/faveurs-aux-hauts-talons/

8. Benjamin, D. (1/08/2013). Voilà les risques que vous prenez à porter des talons hauts.

www.atlantico.fr.https://www.atlantico.fr/decryptage/802901/voilales-risques-que vous-prenez-a-porter-des-talons-hauts-davidbenjamin , consulté le 20/02/2020.

9. Brun, G. (26/09/2016). Conséquences orthopédiques du port des chaussures à talons hauts.

https://aixlesbains.ufcquechoisir.fr/wpcontent/uploads/sites/49/2016/ 12/Cons\%C3\%A9quences-orthop\%C3\%A9diques-du-port-dechaussures-\%C3\%A0-talons-hauts.pdf, consulté le 31/01/2020. 
10. Cnews. (19/10/2016). Quelle est l'origine des talons hauts? https://www.cnews.fr/racines/2016-10-19/quelle-est-lorigine-destalons-hauts-740858, consulté le 31/02/2020.

11. Dave, A.B., Rupel, D., Ross, D., Culle, C., Gallagher, T. et Cohen, A. (Producteurs). (2012-2013). The american real houswives Beverly hills [Série, Saison 3, Episode 2 : The higher heel, the closer to God,].

12. Diallo, A.B. (23/04/2016). Mesdames, voilà les conséquences des talons hauts sur votre santé. https://www.afrikmag.com/voilaconsequences-talons-hauts-sante/, consulté le 31/02/2020.

13. Fresnel, H. (19/01/2016). Mes chaussures, mon miroir. https://www.psychologies.com/Beaute/Style/Articles-et-

Dossiers/Mes-chaussures-mon-miroir

14. Freud, S. (1933). Moise de Michel-Ange. Dans Essais de psychanalyse appliquée (M. Bonapartre et E. Marty, Trad.). Gallimard. (Ouvrage initialement pubilé en 1927.)

15. Freud, S. (1984). La décomposition de la personnalité psychique (RM. Zeitlin, Trad.). Dans Nouvelles conférences d'introduction à la psychanalyse. Gallimard. (Ouvrage initialement publié en 1933.)

16. Freud, S. (1987). Trois essais sur la théorie de la sexuelle (P. Koeppel, Trad.). Gallimard (Ouvrage initialement publié en 1905).

17. Freud, S. (2016). La féminité. Payot. (Ouvrage initialement publié en 1920.)

18. Guéguen, N. (2015). «High heels womens's attractiveness » [Les talons hauts augmentent l'attrait des femmes]. Arch Sex Behav, 44, 2227-2235. Springer. DOI 10.1007/s10508-014-0422-z (Retracted Article)

19. Godelier, M. (1982). La production des grands hommes. Fayard.

20. Heraldie. (20/10/2014). Psychanalyse de la chaussure. heraldie.blogspot.com.

http://heraldie.blogspot.com/2014/10/psychanalyse-de-lachaussure.html

21. Lagache, D. (1961). La psychanalyse et la structure de la personnalité. Dans La psychanalyse. Presses Universitaires de France.

22. Laplanche, J. et Pontalis, J-B. (2019).Vocabulaire de la psychanalyse. Payot. (Ouvrage initialement publié en 1967.)

23. LaPresse. (20/05/2019). La petite histoire des talons hauts. https://www.lapresse.ca/suite/201906/18/01-5230704-la-petitehistoire-des-talons-hauts.php

24. Marie Claire. (s.d.). 20 citations célèbres sur notre amour des chaussures. Https://www.marieclaire.fr/,20-citations-celebres-surnotre-amour-des-chaussures,781609.asp , consulté le 12/05/2021. 
25. Mascret, D. (24/11/2014). La séduction des talons hauts étudiée scientifiquement. https://sante.lefigaro.fr/actualite/2014/11/24/23091seduction-talons-hauts-etudiee-scientifiquement

26. Mormin, G. et Viguier R-J. (1993).Théorie analytique adlérienne. Masson.

27. Mvondo Meka, M. N. (2016). Le symbolisme de la cravate. Dans Revue interdisciplinaire de la faculté des lettres et sciences humaines, numérol6, pp.203-217.

28. Nasio, J.D. (2001). Enseignement de 7 concepts cruciaux de la psychanalyse ( $4^{\mathrm{e} e ́ d}$.). Payot et Rivages. (Ouvrage initialement publié en 1988.)

29. Perez, V. (2018). Supposés symboles de la féminité, les talons hauts étaient à l'origine destinés aux hommes.

https://dailygeekshow.com/talons-hauts-hommes/ , récupéré le 21/01/2020.

30. Porot, A., Porot, M., Sutter, J. et Peliciern Y. (1952/1996). Manuel alphabétique de Psychiatrie. Presses Universitaires de France.

31. Psycho (11, 2017). Nos chaussures et nous. https://posturologie.asso.fr/wp-content/uploads/2017/11/madamefigaro-psycho.pdf

32. Sante.orthodz (21/01/2017). Les talons hauts sont-ils néfastes pour la santé ? http://sante.orthodz.com/2017/01/21/les-talons-hauts-sont-ilsnefastes-pour-la-sante/

33. Talont haut. (20/01/2019). Talon haut. https://fr.wikipedia.org/wiki/Talon_haut, consulté le 02/05/2020.

34. Tourre-Malen, C. (2011). Des chaussures, des talons et des femmes. https://www.cairn.info/revue-ethnologie-francaise-2011-4-page727.htm

35. VonGeschenk, J. (17/02/2015). Histoire de la mode - Les chaussures à https://www.madmoizelle.com/histoire-mode-chaussures-talons322773 